\title{
Impfung in der Schwangerschaft: kritisch hinterfragt
}

\author{
Michael Friedl, Kinderarzt, Heidelberg
}

\section{Impfungen vermitteln ein trügerisches Bild von Sicherheit. Dabei greifen sie gravierend in den gesunden Organismus ein. Und die Liste der mit Impfungen verbundenen Proble- me ist lang. Angesichts der Datenlage muss kritisch hinterfragt werden, ob Schwange- ren eine Impfung empfohlen werden kann.}

Eine Schwangerschaft geht mit vielfältigen Veränderungen einher, die z.B. zu einer stärkeren Durchblutung und Durchlässigkeit der Gewebe sowie deren besserer Ernährung führen. Die glatte Muskulatur wird relaxiert, die Magensäurebildung gesenkt. Die Hypophyse regt die Nebennieren an, vermehrt Kortison, aber auch andere Hormone auszuschütten. Diese Veränderungen schaffen optimale Bedingungen für die kindliche Entwicklung.

Die Auswirkungen auf die immunologischen Zusammenhänge sind allerdings zum größten Teil ungeklärt [45]. Gleiches gilt für Wirkungen des in Impfstoffen enthaltenen Aluminiums auf die durch die hohe Dynamik der Entwicklung sehr empfindlichen Organe in Embryo und Fetus [6]. Damit gleicht eine Impfung in der Schwangerschaft einem Blindflug bzw. einem Experiment.

\section{Risiken der Pertussis-Impfung}

Im Blick auf die Mikrozephaliefälle (vor allem) in Brasilien - die ersten traten im Oktober 2015 auf - ist zu fragen, welchen Einfluss die Diphterie-Tetanus-Keuchhusten-Impfung, die dort seit Oktober 2014 in der 27.-34. SSW verabreicht wird [44], auf den Verlauf der Zikavirus-Infektionen hat.

Die ohnehin unbefriedigende Wirksamkeit des Keuchhusten-Impfstoffs von le- diglich 53-64\% [1,4] bzw. für Kinder im Alter von 2 bis 7 Jahren $41 \%$, zwischen 8 und 12 Jahren sogar nur 24\% [27], scheint in den letzten Jahren noch mehr nachzulassen und führte seit 2012 zu vermehrten Keuchhustenfällen. Es verbreiten sich Untertypen der Keuchhusten-Bakterien, bei denen der aktuelle azelluläre Keuchhusten-Impfstoff (Pa) nicht wirkt. Sein immunologischer Ansatzpunkt ist das Protein Pertactin, das laut Center of Desease Control (CDC) (USA) immer mehr Keuchhusten-Bakterien nicht mehr bilden. Die Forscher werten dies als unmittelbare Anpassung an die geimpfte Umgebung [30]. In jeder Schwangerschaft soll erneut geimpft werden - ungeachtet des Abstands zur letzten [44].

Auch langfristig schafft die azelluläre Keuchhustenimpfung Probleme: Bei Jugendlichen, die von vornherein mit $\mathrm{Pa}$ grundimmunisiert wurden, liegt die Schutzwirkung vier Jahre nach einer Auffrischung im Schulalter bei unter zehn Prozent [23]! Es gibt zunehmend Hinweise darauf, dass eine Keuchhusteninfektion nach vorangegangener (azellulärer) Impfung eine besonders riskante Situation darstellt, da es hierbei (anders als bei nicht geimpften Kindern) zu einer deutlich allergieassoziierten Immunantwort kommt [16]. Dies unterstützt die Beobachtung, dass für diese Kinder ein deutlich erhöhtes Asthma-Risiko besteht.

Angesichts dieser Datenlage spricht auch das Robert-Koch-Institut davon, dass, obwohl die klassischen Keuchhustenerreger nur beim Menschen vorkommen, „aus heutiger Sicht ... eine Eradikation von Pertussis im Gegensatz zu anderen impfpräventablen Krankheiten nicht möglich“ ist. [33]

Die Verschiebung der Erkrankung ins Erwachsenenalter erhöht die Komplikationsrate.

Eine niederländische Studie von 2014 identifizierte vollständig (keuchhusten-) geimpfte Geschwisterkinder als die wichtigste Ansteckungsquelle für Säuglinge in den ersten 6 Lebensmonaten [5]. Diese Ergebnisse führen die Kokonstrategie, die die Pertussisimpfung von Geschwisterkindern empfiehlt, um eine Ansteckung zu vermeiden, komplett ad absurdum. Eine Studie aus dem Jahr 2015 spricht von einem „mehrfach nachgewiesenen Versagen“ der Kokonstrategie $[7,15]$ und erklärt dies mit der Zunahme keuchhustengeimpfter, symptomloser Keuchhustenkranker [2].

Auch die WHO betont ausdrücklich, dass sie die Kokonstrategie nicht empfiehlt: „Neo-natal immunization, and vaccination of pregnant women and household contacts („cocooning“) against pertussis is not recommended by WHO.“ [46]

Eine (Keuchhusten-)Impfung Schwangerer wird von der WHO nicht empfohlen. Brasilien scheint anders beraten. Oder ist es Hilflosigkeit? Wenn ein Prinzip nicht 
funktioniert, wird es zunächst intensiviert: Es muss doch gelingen! Erst, wenn es unausweichlich ist, wird eine faszinierende Idee (hier: Ausrottung einer Krankheit) aufgegeben. Die möglicherweise unbeherrschbare Wandlungsfähigkeit der Natur ist sehr schwer anzuerkennen. Zumal eine Verunsicherung der Bevölkerung hinsichtlich der Richtigkeit des Impfprinzips vermieden werden soll [14]. Dabei ist eine Ausrottung von Krankheitserregern keineswegs gleichzusetzen mit der Ausrottung von Krankheiten.

\section{Risiken der Influenza-Impfung}

„Wir wissen, dass in den USA der Anteil der Grippetodesfälle gleich bleibt, trotz der Tatsache, dass über die Jahre eine immer höhere Zahl von Menschen die Grippeimpfung erhalten haben.“ [20]

1968 führte die CDC eine randomisierte, doppelt-verblindete Studie durch, um den Effekt der Impfung auf die Morbidität und Mortalität zu untersuchen [38]. Die Autoren kamen zu dem Schluss, „dass trotz extensiver Verbreitung des Grippeimpfstoffes ... der Nachweis einer verbesserten Morbidität und Mortalität misslungen ist“.

Bei Patienten über 65 Jahren finden neueste Übersichtsarbeiten keinen Einfluss auf die Sterblichkeit dieser Altersgruppe [32]. Auch die immer wieder behauptete Verminderung von grippeassoziierten Lungenentzündungen lässt sich bei sauberem methodischen Arbeiten nicht nachweisen [18]. Eine Übersichtsarbeit der Cochrane-Collaboration von 2013 findet keinerlei Nutzen der auch in Deutschland immer wieder propagierten Impfung von medizinischem Personal, das ältere Menschen betreut [42]. Um einen Fall von Influenza zu verhindern, müssen mehr als 70 Menschen geimpft werden.

Mindestens ein Drittel der zu Grunde liegenden Studien wurden von der Impf- stoffindustrie gesponsert. Dies relativiert auch die Tatsache, dass keine ernsten unerwünschten Arzneimittelwirkungen (UAW) beobachtet wurden - die Studienautoren sprechen selbst von einer „begrenzten“ Datengrundlage für die Beurteilung der Phänomene [21].

2012/2013 erreichte die Impfung in einer großen Studie eine mittlere Effektivität von gut $20 \%$, wobei im statistischen „Vertrauensbereich“ auch negative Werte enthalten sind, es also durchaus möglich ist, dass die Impfung das Erkrankungsrisiko erhöhte [35].

Die Sicherheit der Grippeschutzimpfung ist - gemessen an ihrer breiten Anwendung - auffallend schlecht untersucht. Jefferson beurteilt die meisten der existierenden Studien als methodologisch schlecht. Sie umfassten meist nur geringe Teilnehmerzahlen und wiesen meist systematische methodische Fehler auf (Selektions-Bias) [19].

Bei Kindern existiert nur eine einzige, ältere Untersuchung mit 35 (!) Teilnehmern. Selbst für Senioren seien insgesamt nur 5 Studien veröffentlicht mit einer Laufzeit von jeweils maximal 7 Tagen und insgesamt 3000 Teilnehmern.

Angesichts dieser Situation ist umso erschreckender, dass so viele und verschiedenartige UAWs im zeitlichen Zusammenhang mit der Influenzaimpfung aktenkundig sind: Sie erhöht das Risiko, an autoimmunologischer Gefäßentzündung (Riesenzellarteriitis) oder rheumatoider Polymyalgie [40] zu erkranken oder am Churg-Strauss-Syndrom (Asthma und systemische autoimmunologische Gefäßentzündung) [10].

Das Gesamtrisiko, nach einer Grippeimpfung an einem sog. Guillain-Barré-Syndrom (GBS) zu erkranken, ist um mindestens den Faktor 4 [31], für eine schwere Verlaufsform des GBS sogar 8-fach erhöht [13]. Insgesamt ist das GBS eine der häufigsten schweren Komplikationen des Grippeimpfstoffes [8, 9, 12,31,41]. 
Sogenannte demyelinisierende Erkrankungen, bei denen die Markscheiden der Nervenzellen im Gehirn und Rückenmark zerstört werden (wie die Akute Demyelinisierende Enzephalomyelitis ADEM -, eine der Multiplen Sklerose verwandte, akute Erkrankung) treten immer wieder in engem zeitlichem $\mathrm{Zu}$ sammenhang zur Grippeimpfung auf $[3,11,25,26,36,37]$.

Auch bei Kindern werden immunologische Entzündungen des Sehnerven beschrieben - diese sind häufig ein Erstsymptom einer Multiplen Sklerose [24]; Erblindung durch Schädigung des Sehnerven [22]; auch die Schädigung anderer Hirnnerven [17]. Neurologische Komplikationen wie Hirnentzündung (Enzephalitis) [43] oder Transverse Myelitis, eine mit Lähmungen einhergehende Entzündung des Rückenmarks [29].

Die Liste ließe sich fortsetzen (siehe http://www.impf-info.de/die-impfungen/grippe/140-grippe-die-impfung. html, Dr.Steffen Rabe).

\section{Diskussion und Fazit}

Kann man bei dieser Datenlage, die sicher nicht alle bestehenden Probleme umfasst, empfehlen, Schwangere zu impfen?

Karin Mölling, eine renommierte Virusforscherin, kommt nach Abschluss ihrer beruflichen Karriere zu der Auffassung, dass Viren und Bakterien per se dem Menschen nichts anhaben können. Er lebe mit ihnen seit Jahrtausenden in friedlicher Koexistenz, habe sie im wahrsten Sinne verinnerlicht. Nur ein in seinen Grundbedürfnissen verletzter und dadurch aus der Balance geratener Organismus biete ihnen Gelegenheit zur Vermehrung.

„Der Mensch ist ein (...) komplexes Ökosystem (...) in diesem (...) herrscht kein (...) Krieg, (...) sondern eine Balance (...). Wenn äußere Einflüsse die Balance zerstören(,) meistens ist der Mensch selbst der Verur- sacher (...) entstehen Krankheiten. Viren und Bakterien sind (...) Nutznießer von (...) Schwächen des Wirts“. [28]

Daher kann man (Entzündungs-)Krankheiten auch als Indikatoren für ins Ungleichgewicht geratene geistig-seelischkörperliche Zusammenhänge betrachten. Dann sind Krankheiten nicht der Verlust oder ein Unterliegen im Kampf um die Gesundheit, sondern eine autonome Anstrengung des menschlichen Organismus, zu einer verbesserten Gesundheitslage zu kommen. Der Blick wird auf die persönlich beeinflussbaren und zu gestaltenden Lebensumstände gelenkt.

Da Impfungen in einen gesunden Menschen eingreifen, gilt es, individuell Vorund Nachteile aufzuspüren und sich zu entscheiden. Allgemeine Zwangsmaßnahmen führen nicht zur Verantwortung des Einzelnen für seine Gesundheit und die Gemeinschaft.

Die Haltung des Berufsverbandes der Kinderärzte, der von seinen Mitgliedern fordert, die Empfehlungen der STIKO ohne individuelle Abweichungen in Umfang und Zeitpunkt umzusetzen [14], führt dazu, dass Fragen nach Nebenwirkungen beantwortet werden mit: „Die gibt es nicht.“

Dabei hat der Bundesgerichtshof anders geurteilt:

„Entscheidend für die ärztliche Hinweispflicht ist nicht ein bestimmter Grad der Risikodichte, insbesondere nicht eine bestimmte Statistik. Maßgebend ist vielmehr, ob das betreffende Risiko dem Eingriff spezifisch anhaftet und es bei seiner Verwirklichung die Lebensführung des Patienten besonders belastet (BGHZ 126, 386 ff. (389)). Der Senat hält deshalb daran fest, dass grundsätzlich auch über äußerst seltene Risiken aufzuklären ist.“ [34]

Die Impfung mit einem „Recht auf Gesundheit" (gibt es das wirklich?) gleichzusetzen und Eltern abzusprechen, ihren Kindern dieses „Recht zu verweigern“ [14], schafft eine Diskriminierung und
Stigmatisierung von Personen, die ihr persönliches Recht in Anspruch nehmen.

Wenn Eltern berichten, dass selbst bei schwersten Erkrankungen unmittelbar nach einer Impfung behandelnde Ärzte einen Zusammenhang kategorisch ausschließen, wird deutlich, dass ein trügerisches Bild von Sicherheit vorhanden ist. Hier werden gesetzliche und wissenschaftliche Grundsätze missachtet. Solche Fälle müssten im Sinne der Arzneimittelsicherheit an das Paul-EhrlichInstitut (PEI) gemeldet werden. Das kann mittlerweile auch jeder Laie tun.

Jeder Arzt ist primär seinem Patienten verpflichtet. In Zeiten einer alles beherrschenden Wirtschaft, die mit tausenden Lobbyisten in Deutschland Einfluss auf Entscheidungsträger nimmt, sollten Entscheidungen jedes Expertengremiums hinsichtlich der Lauterkeit ihrer Beweggründe und eventueller individueller Verflechtungen hinterfragt werden.

\section{Literatur}

Die Literatur ist in der Online-Version der Zeitschrift Die Hebamme verfügbar unter www.thieme-connect.de/products.

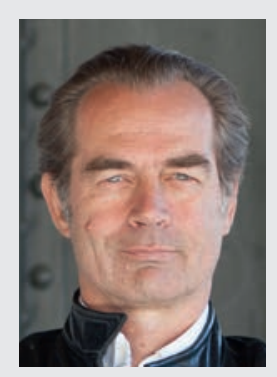

Michael FriedI

Facharzt für Kinderund Jugendmedizin Vorsitzender Ärzte für individuelle Impfentscheidung e.V.

Dossenheimer Landstr. 37 69121 Heidelberg E-Mail: praxis@michaelfriedl.de www.individuelleimpfentscheidung.de 


\section{Literatur}

1 Ad hoc group for the study of pertussis vaccine. Placebo-controlled trial of two acellular pertussis vaccines in Swedenprotective efficacy and adverse events. Lancet 1988; 1: 955-960

2 Althouse BM, Scarpino SV et al. Asymptomatic transmission and the resurgence of Bordetella pertussis. BMC Medicin (2015) 13: 146

3 Arai $\mathrm{M}$ et al. Acute disseminated encephalomyelitis following influenza vaccination: report of a case with callosal disconnection syndrome. Rinsho Shinkeigaku 2014; 54 (2): 135-9

4 Baxter R et al. Effectiveness of pertussis vaccines for adolescents and adults: case-control study. BMJ 2013; 347: f4249

5 Bertilone $\mathrm{C}$ et al. Finding the who' in whooping cough: vaccinated siblings are important pertussis sources in infants 6 months of age and under. Commun Dis Intell Q Rep 2014; 38 (3): E195-200

6 BGM-Studie summary2014: http:// www.bmg.gv.at/home/Aluminium_Toxikologie

7 Castagnini LA, Healy et al. Impact of Maternal Postpartum Tetanus and Diphtheria Toxoids and Acellular Pertussis Immunization on Infant Pertussis Infection. Clin Infect Dis 2012; (1): 78-84

8 Choe, YJ et al.Serious adverse events following receipt of trivalent inactivated influenza vaccine in Korea, 2003-2010. Vaccine 2011; 29 (44): 7727-32

9 Finch NA et al. Guillain-Barré syndrome in an older man following influenza vaccination. J Am Pharm Assoc (2003). 2014; 54 (2): 188-92

$10 \mathrm{Fu} \mathrm{MH}$ et al. Churg-Strauss Syndrome Following Vaccination Against 2010 Influenza A (H1N1): A Case Report. Acta Neurol Taiwan 2014; 23 (3): 95-101

11 Fujii K et al. Acute disseminated encephalomyelitis following 2009 H1N1 influenza vaccine. Pediatr Int 2012; 54 (4): 539-41

12 Galeotti F et al. Risk of Guillain-Barré syndrome after 2010-2011 influenza vaccination. Eur J Epidemiol 2013; 28 (5): 433-44
13 Geier MR, Geier DA, Zahalsky AC. Influenza vaccination and Guillain Barre syndrome. Clin Immunol 2003; 107: 116-121

14 Hartmann W. Kinder- und Jugendarzt 46 (2015) 4 S.15

15 Healy CM et al. Evaluation of the impact of a pertussis cocooning program on infant pertussis infection. Infect Dis J 2015; 34 (1): 22-26

16 Hendrikx LH et al. Different IgG-subclass distributions after whole-cell and acellular pertussis infant primary vaccinations in healthy and pertussis infected children. Vaccine 2011; 29 (40): 6874-80

17 Ishii K et al. Glossopharyngeal nerve and vagus nerve palsies associated with influenza vaccination. Intern Med 2014; 53 (3): 259-61

18 Jackson ML et al. Influenza vaccination and risk of community-acquired pneumonia in immunocompetent elderly people: a population-based, nested case-control study. Lancet 2008; 372: 398-405

19 Jefferson T. Influenza vaccination: policy versus evidence. BMJ 2006; 333; 912-915

20 Jefferson T. Neuraminidase inhibitors for preventing and treating influenza in healthy adults: systematic review and meta-analysis. Cochrane Database Systematic Review, 23 April 2009

21 Jefferson $T$ et al. Vaccines for preventing influenza in healthy adults. Cochrane Database Syst Rev 2014; 3: CD001269

22 Kawasaki A et al. Bilateral anterior ischemic optic neuropathy following influenza vaccination. J NEUROOPHTHALMOL 1998; 18 (1): 56-9

23 Klein N et al. Kaiser Permanente Study Finds Effectiveness of Routine Tdap Booster Wanes in Adolescents. Pediatrics 2016

24 Korematsu S et al. Elevated serum anti-phosphatidylcholine IgG antibodies in patients with influenza vaccinationassociated optic neuritis. Vaccine 2014; 32 (48): 6345-8

25 Maeda $\mathrm{K}$ et al. Acute disseminated encephalomyelitis following 2009 H1N1 influenza vaccination. Intern Med 2012; 51 (14): 1931-3
26 Machicado JD et al. Acute disseminated encephalomyelitis following seasonal influenza vaccination in an elderly patient. Clin Vaccine Immunol 2013; 20 (9): 1485-6

27 Misegades LK. Estimating the Effectiveness of Acellular Pertussis Vaccines. Clin Infect Dis 2012; 55 (10): 1432-1433. doi: 10.1093/cid/cis66

28 Mölling K. Supermacht des Lebens. 2015 C.H.Beck München, S14-5

29 Nakamura et al. Neurologic complications associated with influenza vaccination: two adult cases. Intern Med 2003; 42: 191-4

30 Pawloski LC et al. Prevalence and molecular characterization of pertactindeficient Bordetella pertussis in the US. Clin Vaccine Immunol 2013; doi: 10.1128/CVI.00717-13

31 Prestel J et al. Risk of Guillain-Barré syndrome following pandemic influenza $A(H 1 N 1) 2009$ vaccination in Germany. Pharmacoepidemiol Drug Saf 2014; 23 (11): 1192-1204

32 Rizzo C et al. Influenza-related mortality in the Italian elderly: no decline associated with increasing vaccination coverage. Vaccine 2006; 24 (42-43): 6468-75

33 RKI. Pertussis - Stand 2010

34 RKI. Hinweise für Ärzte zum Aufklärungsbedarf über mögliche unerwünschte Wirkungen bei Schutzimpfungen/Stand: 2007. Epidemiologisches Bulletin 2007; 22; 25: 209

35 Rondy $\mathrm{M}$ et al. 2012/13 INFLUENZA VACCINE EFFECTIVENESS AGAINST HOSPITALISED INFLUENZA A(H1N1) PDM09, A(H3N2) AND B: ESTIMATES FROM A EUROPEAN NETWORK OF HOSPITALS Euro Surveill. 2015; 20 (2): pii=21011

36 Sacheli A et al. Influenza vaccine-induced CNS demyelination in a 50-year-old male. Am J Case Rep 2014; 15: 368-73

37 Shoamanesh A. Acute disseminated encephalomyelitis following influenza vaccination Vaccine. 2011; 29 (46): 8182-5

38 Schoenbaum SC, Mostow SR et al. Studies with Inactivated Influenza Vaccines Purified by Zonal Centrifugation. Bull World Health Organ 1969; 41: 531-535 
39 Shimizu M et al. Relapse of systemic juvenile idiopathic arthritis after influenza vaccination in a patient receiving tocilizumab. Clin Vaccine Immunol 2012; 19 (10): 1700-2

40 Soriano A, Lupus et al. Giant cell arteritis and polymyalgia rheumatica after influenza vaccination: report of 10 cases and review of the literature. 2012; 21 (2): 153-7

41 Souayah N et al. Guillain-Barré syndrome after influenza vaccination in the United States, a report from the CDC/FDA vaccine adverse event reporting system (1990-2009). Neuroepidemiology 2012; 38 (4): 227-32

42 Thomas RE et al. Influenza vaccination for healthcare workers who care for people aged 60 or older living in long-term care institutions. Cochrane Database Syst Rev 2013; 7: CD005187

43 Turkoglu R et al. Brainstem encephalitis following influenza vaccination: favorable response to steroid treatment. Vaccine 2009; 27 (52): 7253-6

44 Vranjac A. Governo Do Estado Secretaria De Estado Da Saúde, Coordenadoria De Controle De Doenças, Centro De Vigilância Epidemiológica. Informe Técnico Vacina Difteria, Tétano e Coqueluche dTpa. Outubro de 2014

45 Weyerstahl T, Stauber M. Gynäkologie und Geburtshilfe; Georg Thieme Verlag KG Stuttgart; 4. Aufl. 2013

46 WHO. Recommendations for routine immunization. (Abruf 20.02.2016) 